DOI: https://doi.org/10.46296/yc.v5i9ucedespsoct.0126

\title{
PLAN DE MEJORA PARA EL SERVICIO DE VINOS EN EL RESTAURANTE NO ESTATAL "EL PASO" DE LA CIUDAD CAMAGÜEY
}

\section{IMPROVEMENT PLAN FOR THE WINE SERVICE IN THE NON-STATE RESTAURANT "EL PASO" IN THE CITY OF CAMAGÜEY}

\author{
Cruz-Carbonell Mérida Esmunda ${ }^{1}$; Zambrano Jessica Mariela ${ }^{2}$; \\ Jiménez-Rodríguez Adys ${ }^{3}$; Guerra-Vázquez Lizbet ${ }^{4}$
}

${ }^{1}$ Profesor Asistente del departamento Tecnología y Ciencias de los Alimentos de la Universidad de Camagüey "Ignacio Agramonte Loynaz". Camagüey, Cuba. Correo: merida.cruz@reduc.edu.cu. ORCID ID: https://orcid.org/0000-0001-9283-1108

${ }^{2}$ Docente investigadora de la Universidad Laica Eloy Alfaro de Manabí ULEAM. Manta, Ecuador. ORCID ID: https://orcid.org/0000-0003-1370-5934

${ }^{3}$ Departamento de Asesoría Jurídica de la Universidad de Camagüey "Ignacio Agramonte Loynaz". Camagüey, Cuba. Correo: adys.jimenez@reduc.edu.cu. ORCID ID: https://orcid.org/0000-0002-2515-8336

${ }^{4}$ Oficina Territorial de Normalización de Camagüey (OTN). Camagüey, Cuba. Correo: lisazul@gmail.com. ORCID ID: https://orcid.org/0000-0001-6675-8250

\section{Resumen}

Este trabajo se realiza con el objetivo de elaborar un plan de mejoras en el servicio de vinos que permita su integración a los productos ofertados en el restaurante "El Paso". Este restaurante de la Ciudad de Camagüey es uno de los preferidos dentro del sector no estatal; sin embargo, no ha incorporado el servicio especializado de vinos como elemento integrado a su oferta. La aplicación de métodos teóricos y empíricos utilizados permitieron dar respuesta a la problemática planteada e hicieron posible visualizar el papel del vino como valor agregado a los productos. Como resultado fueron identificados los problemas y áreas claves relacionadas con el servicio de los vinos, lográndose la implementación de mejoras para efectuar la incorporación gradual, pautada, orgánica y procesual de este servicio especializado, lo que aporta elegancia y profesionalidad a la oferta, satisfacer los gustos y preferencias del cliente, elevando los estándares de calidad, con la integración del servicio de vinos a la cartera de productos que ofrece el restaurante. La elaboración del plan de mejoras parte de un grupo de pasos que, regidos por una metodología, han recogido desde los resultados directos arrojados por el diagnóstico hasta la proyección de un nuevo estado deseado, con su respectivo diseño de objetivos y acciones por áreas significativas de intervención.

Palabras claves: plan de mejora, vinos, servicio de vino, restaurante.

\begin{abstract}
This work is carried out with the aim of preparing a plan for improvements in the wine service that allows its integration into the products offered in the "El Paso" restaurant. This restaurant in the City of Camagüey is one of the favorites within the non-state sector; however, it has not incorporated the specialized wine service as an integrated element in its offer. The application of theoretical and empirical methods used allowed to respond to the problem raised and made it possible to visualize the role of wine as an added value to the products. As a result, the problems and key areas related to the wine service were identified, achieving the implementation of improvements to carry out the gradual, guided, organic and procedural incorporation of this specialized service, which brings elegance and professionalism to the offer, satisfying the tastes
\end{abstract}

Información del manuscrito:

Fecha de recepción: 26 de julio de 2021.

Fecha de aceptación: 29 de septiembre de 2021.

Fecha de publicación: 11 de octubre de 2021. 
and preferences of the customer, raising quality standards, with the integration of wine service to the portfolio of products offered by the restaurant. The preparation of the improvement plan is based on a group of steps that, governed by a methodology, have collected from the direct results obtained by the diagnosis to the projection of a new desired state, with their respective design of objectives and actions for significant areas of intervention.

Keywords: improvement plan, wines, wine service, restaurant.

\section{Introducción}

El turismo cultural urbano sustentable representa una alternativa para el desarrollo económico, social y cultural de las comunidades urbanas, para lo cual es necesario desarrollar productos y servicios turísticos apropiados.

Mantener este reto implica realizar estudios constantes de las particularidades de su propio desarrollo; y a decir de los especialistas ha de hacerse desde un enfoque multidisciplinario e integral, dada la complejidad de este fenómeno que conlleva implicancias no sólo económicas y medioambientales, sino también políticas que afectan a toda la sociedad, no sólo a los que trabajan en turismo. (Sánchez, K., 2012)

Desde finales del año 2010 el Estado Cubano comenzó en el país un proceso de transformaciones que convirtió la batalla económica en la tarea principal y en la clave para la sostenibilidad y preservación de dicho sistema social.

En la actualidad las empresas turísticas avanzan hacia un camino mucho más profesional, en el que cada detalle cuenta, de ahí la importancia de la calidad de este producto.

El presente trabajo se realiza en la ciudad de Camagüey, localidad que ha experimentado un crecimiento importante en el número de restaurantes vinculados al sector no estatal, los cuales luchan a su vez por escalar en la preferencia tanto de visitantes nacionales como extranjeros.

Un pequeño número de estos restaurantes han incursionado en determinados servicios especializados, logrando tales estándares de calidad que han resultado válidos, a través de contratos con las principales agencias de viajes de Cuba, para formar parte de los circuitos de 
recorrido, frecuentemente visitados en el caso de Camagüey por turistas alemanes, argentinos, suecos y estadounidenses.

La gastronomía es considerada como la identidad de cada provincia, región o país, la cual, mediante la elaboración de su comida típica, da a conocer a sus visitantes, la riqueza de su cultura y sobre todo de los sembríos, frutas y fauna que posee, haciendo sentir al turista parte de la cultura de dicho lugar. Carvache, M., Carvache, W., Orden, M., Macas, C., (2017), citado por Rentería, S. (2017).

La explosión de visitas turísticas que acuden a estas instituciones estatales y no estatales, promovidas a su vez por las políticas del ministerio y el gobierno local, ha generado nuevas necesidades relacionadas con la cadena de suministros, operaciones logísticas, capacitación del personal, mejoras en los procesos de comercialización, acciones todas en función de lograr una de las estrategias de primordial importancia para el desarrollo del turismo en Cuba: mejorar la calidad de los servicios medida por la satisfacción de los clientes y ajustada a los estándares internacionales. (Castro, Y., 2015)

En las normas ISO 9000 (2016) se plantea que la organización deberá mejorar de manera constante la eficacia del sistema, significa que no se tiene que implementar ningún proceso adicional, sino revisar la correlación entre los procesos, asegurándose de que ayuden juntamente a la mejora constante.

Cuando se revisan estos aspectos, debatidos con objetividad en Simposios Internacionales sobre Comercio y los Servicios desarrollados, se percibe la presencia de dificultades de manera particular en los servicios y más evidentes en algunas instalaciones del sector no estatal con apoyo al turismo. (Castro, Y., 2015)

Tal es el caso del restaurante "EI Paso", el cual exhibe un porciento significativo de visitantes, sin embargo, se registran algunas dificultades que giran alrededor de lo siguiente:

- El servicio del vino se experimenta como un aspecto aislado, separado y circunstancial a las ofertas gastronómicas 
- Los productos que se ofertan no cuentan con el valor agregado que puede aportar la cultura del vino, se percibe poco dominio de esto en los dependientes gastronómicos

fundamentalmente.

- Desnivel en cuanto a las expectativas de los clientes respecto al servicio del vino (lo mismo aparecen clientes que esperan encontrar un servicio de vino, como clientes que se sorprenden de allí encontrar algunos vinos de calidad).

- Cierta desproporción en cuanto a la promoción del vino y su modo de servicio.

De lo expuesto se resume que existen carencias en el servicio del vino percibidas como falta de proactividad para generar mejoras en los productos ofertados en el establecimiento, en lo particular vinculadas con la cultura que gira alrededor de su servicio; aspecto que no permite incrementar la satisfacción de los clientes, al menos por este concepto, de una forma estable.
El vino como bebida acompañante se lo puede encontrar en establecimientos cuyos productos se destacan en servir carnes, ya sean asadas o al carbón, dependiendo del lugar, la categoría y su calidad en el servicio, se puede encontrar un vino cuyo sabor y marca sean de calidad. (Rentería, S., 2017)

La hospitalidad y la atención personalizada se encuentran estrechamente ligadas a un diálogo constante con el cliente y a una especialización que convierta su estancia en una experiencia única en su conjunto (Boné, Rey, Fusté, 2015).

Por lo antes expuesto se realiza este trabajo con el objetivo: Elaborar un plan de mejoras en el servicio de vinos que permita su integración a los productos ofertados en el restaurante "El Paso" y contribuya a la satisfacción de los clientes.

\section{Metodología (Materiales y métodos)}

En la ciudad de Camagüey se ha creado un grupo de restaurantes particulares, llamados también paladares, casas renta, 
aprovechando la coyuntura económico social del país, tal es el caso del restaurante "El Paso", el cual se ha escogido como objeto de estudio porque se aviene a lo que se ha explicado hasta el momento y es uno de los restaurantes del sector no estatal que, a pesar de su corta experiencia, ha dado empuje al turismo que se desarrolla actualmente en la ciudad.

El restaurante "El Paso" se encuentra ubicado en la Plaza El Carmen, fue fundado en el año 2013; está ubicado en el interior de una casa tipo colonial, rasgos que caracterizan toda la vecindad, próximo a la iglesia El Carmen. Se encuentra rodeado por el complejo escultórico compuesto por figuras de bronce en tamaño natural, que representan la vida cotidiana de la ciudad, como son; el lector de periódico, el aguatero, los amantes, y las chismosas; todos realizados por la artista Marta Jiménez, quien expone su casa como estudio-taller al frente del propio restaurante.

Desde su inauguración comienza a ofrecer servicios de alimentos $y$ bebidas y posee un salónrestaurante, un bar, un patio, una terraza, así como un reservado climatizado para la ejecución de tales servicios, en los horarios de 10.00 am a 11.00 pm diariamente.

Su cocina es internacional, aunque se resaltan platos tradicionales cubanos y locales. establecimiento cuenta con un total de 14 trabajadores, de ellos 6 dependientes, 4 cocineros, 2 jefes de turnos, y un administrador, además de la dueña del establecimiento.

En este sentido, la actividad de restauración se puede resumir como la conversión de los factores de producción (alimentos, capital, trabajo) mediante la producción y el servicio, en productos solicitados por un cliente que busca el consumo, la satisfacción y el bienestar. (Parra, F., Vasallo, V., 2016)

Asumiendo la definición anterior y analizando las carencias y los datos presuntivos los autores deciden proseguir en la investigación escogiendo pasos metodológicos que han dividido en dos fases 0 momentos: Un 1er momento o fase de profundización en el diagnóstico y una 2da, compuesta por los pasos para el diseño del plan de mejoras. 
Relacionado con el plan de mejoras Aguilar, J. (2016) expresa, que la mejora continua se describe al hecho de que nada puede pensarse como algo terminado o mejorado en forma conclusiva, todo es un proceso de cambio, de desarrollo y con potencialidades de mejora continua. La existencia humana no es estática, sino un proceso dinámico en constante evolución, como porción de la naturaleza universal. Este juicio es aplicable tanto a personas, organizaciones y sus actividades.

El diagnóstico del estado actual del proceso del servicio del vino en el restaurante no estatal "El paso" se realizó conforme a los pasos siguientes:

1.- Análisis documental de registros oficiales del propio restaurante previa solicitud de autorizo por parte del dueño del establecimiento. A saber: cartas menú, reportes de ingresos, registros estadísticos de clientes en aras de identificar los mercados que frecuentan el establecimiento, encuestas de satisfacción, si existen.

2.- Definición de indicadores sujetos a medición y valoración a través de la aplicación de métodos y técnicas.

3.- Recopilación de información a través de la aplicación de encuestas a clientes y entrevistas a trabajadores y administradores del establecimiento, y su posterior análisis de resultados para constatar la idea a defender en el trabajo de investigación. Se incluye encuesta de satisfacción a los clientes (nacionales y extranjeros) por no contar con estos resultados cuando se realizó el análisis de documentos existentes en el establecimiento.

4- Comparación entre la situación actual y el estado deseado sobre la prestación del servicio de vinos a partir de la elaboración de una Matriz DAFO.

- Este paso es clave para la confección de la propuesta pues se basa en el principio de convertir las amenazas en oportunidades y la atenuación de las debilidades buscando apoyo en las fortalezas. 
1. Estudio de documentos propios del restaurante, análisis derivados.

- Se analizaron algunos ejemplares de cartas menú, revisando elementos tales como: la confección, estructura, modo de presentación de las mismas y si tienen en existencia o no cartas de vino. La confección de las cartas analizadas tiene calidad en cuanto a sus modos de presentación, tienen un formato prediseñado digitalmente con un balance de elementos, la estructura es bastante adecuada a lo que se exige para su confección y son prototipo de la oferta que generalmente invita a una variedad de platos factibles a maridar, se constata en cambio que no existen cartas de vino.

2.- Análisis del comportamiento de los indicadores reflejados en las encuestas y entrevistas realizadas.

Se aplicaron encuestas al $100 \%$ del personal de servicios (6), donde fueron analizados los siguientes aspectos:

- $\quad$ El primer indicador: Nivel de conocimiento acerca de los elementos del vino; se midió a través de escalas: alto, medio, bajo y nulo.

- El segundo indicador: Grado en que ha sido identificado del tema como necesidad para mejorar los servicios.

- Indicador: nivel de posibilidades de integración del servicio del vino a los productos que se ofertan y los mercados que lo solicitan.

- Indicador: disposición para asimilar el plan de mejoras con inclusión del servicio de vinos.

Con respecto a los resultados de las entrevistas realizadas.

Se aplicaron al jefe del establecimiento y demás personal administrativo (4 en total). Los resultados que se obtuvieron fueron similares a los de las encuestas:

- Primer indicador: preparación del personal sobre la cultura del vino.

- Segundo indicador: importancia del tema para introducir servicios especializados, 
- Indicador: posibilidades de integración del servicio del vino a los productos que se ofertan y según mercados que lo solicitan.

- Indicador: disposición para introducir en lo adelante mejoras en los servicios del restaurante, a partir de servicios especializados como lo es el servicio del vino.

Dado que en el restaurante objeto de estudio no se aplican encuestas de satisfacción a los clientes, los investigadores deciden realizar encuestas a algunos clientes, escogidos de manera aleatoria para explorar sus expectativas respecto a los servicios y productos que ofrece el restaurante y sus niveles de satisfacción.

Por lo expuesto se hace necesario medir la calidad de los servicios para que los administrativos conozcan como lo evalúan sus clientes. En este sentido, Monroy, M., Urcádiz, F. (2018) refiere que la realización de encuestas para evaluar los impactos de procedimientos para la gestión de procesos de restaurantes revelando la necesidad de realizar un análisis estratégico del servicio para los empresarios.
En las encuestas se tomaron en cuenta los siguientes aspectos:

a) Variedad de platos

b) Variedad de vinos ofertados

c) Relación calidad precios de los vinos en el restaurante.

d) Eficiencia del servicio de vinos (incluye presencia de: dependiente, de accesorios e instrumentos, del ritual)

e) Temperatura de los vinos

f) Sugerencias de maridajes

Se encuestaron un total de 41 comensales, de ellos, 21 cubanos y 20 extranjeros de diversas procedencias: 10 canadienses, 4 españoles, 3 italianos, 3 argentinos.

Los resultados recogidos a través de índices evaluativos de: Excelente, Bien, Regular y Mal.

Para ahondar más en el diagnóstico y poder realizar una comparación entre la situación actual y el estado deseado sobre la prestación del servicio de vinos en el restaurante objeto de estudio, se aplicó un Análisis DAFO con los principales responsables del establecimiento. 
Esta es una tarea para activar la actividad mental al igual que la lluvia de ideas, con la ventaja sobre la anterior de que va entrenando a los participantes en la búsqueda de soluciones ante aquellas barreras (de tipo psicológica, administrativa u otras) que puedan presentarse, fundamentalmente en las amenazas y debilidades que no dejan ver las perspectivas del arte de combinar la degustación de alimenticios con la realización de un servicio especializado de vino.

3.- Análisis comparativo a partir de la Matriz DAFO

Para realizar esta comparación entre la situación actual del problema y el estado deseado, visualizado con más objetividad a través de la elaboración de una Matriz DAFO, primeramente, deben analizarse los escenarios internos y externos que rodean al establecimiento objeto de estudio, a este primer paso se le llama Análisis DAFO

\section{El Análisis DAFO permitió} determinar las ventajas competitivas de la instalación, bajo el análisis de emplear las soluciones que más le convenga a la entidad objeto de estudio, en función de sus características propias y de las del mercado en que se mueve.

En otro orden también facilitó el análisis de situaciones que representan amenazas para la organización y por tanto pueden agravar sus problemas, las cuales se convierten en factores externos que hay que controlar, enfrentar y defenderse.

\section{Resultados (análisis e interpretación de los resultados)}

Al aplicar las encuestas al $100 \%$ del personal de servicios (6), se obtuvieron los siguientes resultados:

- Con respecto al indicador: Nivel de conocimiento acerca de los elementos del vino; se midió a través de escalas: alto, medio, bajo y nulo. De manera general, los resultados no fueron satisfactorios, siendo escogidos para los 6 trabajadores implicados directamente al servicio (dependientes) los niveles bajo y nulo (4 bajos y 2 nulos). Se recogieron referencias además sobre la insuficiente capacitación de este personal, reflejada en las 
vías de formación diferentes: 2 que se han formado en Formatur y 4 en escuelas de comercio y gastronomía, donde el rigor en cuanto a la preparación es menor

- El segundo indicador: Grado en que ha sido identificado del tema como necesidad para mejorar los servicios, se comporta como bajo para el $100 \%$ de los trabajadores de servicio encuestados. En el momento de iniciada la investigación no se veía la importancia del tema para enriquecer los servicios e incrementar los ingresos, percatándose un mejor nivel de percepción sobre la introducción del servicio del vino para la mejora de los servicios generales del restaurante, después de la intervención del investigador.

- Sobre el nivel de posibilidades de integración del servicio del vino a los productos que se ofertan y los mercados que lo solicitan. El $90 \%$ de los empleados encuestados (5) considera que las posibilidades reales son bajas, saliendo a relucir como limitantes, los altos precios del vino y la carencia de un seguimiento a la tipología de clientes que visita el restaurante, en particular por desconocimiento de la importancia que esto tiene para satisfacer sus gustos $y$ preferencias respecto al consumo de alimentos y bebidas.

- Sobre la disposición para asimilar el plan de mejoras con inclusión del servicio de vinos: En este indicador las respuestas del $100 \%$ de la muestra es alto, a partir de la visualización de posibilidades que ofrece la investigación y las perspectivas de superación para el despliegue de una actividad más eficiente.

Con respecto a los resultados de las entrevistas realizadas:

Se aplicaron al jefe del establecimiento y demás personal administrativo (4 en total). Los resultados que se obtuvieron fueron similares a los de las encuestas:

- En su primer indicador, se reconoce que de manera general no existe una cultura del vino y que el personal de servicio contratado nunca ha recibido un curso de sommelier, por otro lado, el índice de preparación de los que proceden de las escuelas de 
comercio es inferior que el de los que proceden de Formatur, siendo estos últimos la minoría. Los niveles se comportan como sigue: Nulo (25\%), Bajo (65\%) y Alto $(10 \%)$

De igual manera se desconocen estudios sobre merchandising, técnicas de ventas, comportamiento de mercados, ni se efectúa control de cifras sobre visitantes que acude al establecimiento tanto emitidos por las agencias a través de la inclusión del restaurante en el circuito de recorrido, como de los que visitan la instalación espontáneamente. Someramente se conoce que la mayor cantidad de turistas que visitan la provincia siguen siendo canadienses.

- Con respecto a la importancia del tema para introducir servicios especializados, 3 de los 4 entrevistados admiten haber pensado en esta necesidad, pero nunca tomaron acciones en pos de ellas por la implicancia que tiene en las inversiones y gastos para el negocio, sumado a la falta de preparación para asumir el reto. Tímidamente se introducen algunos vinos, que en general son escogidos por los precios asequibles más que por sus marcas, procedencia emergencias de optimización según demandas de mercados.

- Sobre las posibilidades de integración del servicio del vino a los productos que se ofertan y según mercados que lo solicitan: En la actualidad las posibilidades reales del establecimiento son percibidas como bajas para los 4 entrevistados, dado los elementos reflejados anteriormente. Se agrega como elemento de peso también el insuficiente conocimiento sobre el maridaje, refieren que los hizo colocar el servicio del vino en una posición de lujo y no como parte de un elemento habitual en la oferta de cualquier comensal, independientemente de su mercado de procedencia.

- Por último, se evalúa de alta la disposición para introducir en lo adelante mejoras en los servicios del restaurante, a partir de servicios especializados como lo es el servicio del vino (100\%). A partir de la intervención del investigador se percibe una postura alentadora respecto al 
desembarazo con el que se piensan asumir los nuevos retos, como la construcción de una cava.

Resultados de las encuestas de satisfacción a los clientes, para explorar sus expectativas respecto a los servicios y productos que ofrece el restaurante y sus niveles de satisfacción.

Se encuestaron un total de 41 comensales, excluyendo a menores de 16 años por razones obvias; de ellos, 21 cubanos y 20 extranjeros de diversas procedencias: 10 canadienses, 4 españoles, 3 italianos, 3 argentinos.

Los resultados recogidos a través de índices evaluativos de: Excelente, Bien, Regular y Mal, se comportaron de la siguiente forma:

-Indicador a): Evaluado entre Excelente y Bien la variedad de platos $100 \%$ para ambos tipos de clientes

-Indicador b): Evaluadas de Regular las ofertas de vino (82\%), para un $8 \%$ de satisfacción entre extranjeros. Para los cubanos, un $35 \%$ evalúa la oferta de vinos como Bien e incluso un $40 \%$ lo sitúa como Excelente.
-Indicador c): Bien para el $93 \%$ de extranjeros, Regular para los cubanos, precios evaluados como muy altos: $72 \%$ para estos últimos.

-Indicador d): Baja para los extranjeros (Regular un 75\% y mal $20 \%$ ). Para los cubanos, percibida como Bien $55 \%$ y $25 \%$ lo evalúa como Excelente, con una cifra restante de abstenciones en el resto de los niveles.

-Indicador e): Bien para el 100\% de los encuestados (nacionales y extranjeros)

-Indicador f): Mal para el $45 \%$ de los comensales extranjeros, Regular para un 30\%; entre cubanos evaluadas de Bien, $45 \%$, con cifra alta de abstenciones.

A modo de resumen, el nivel de satisfacción de los clientes se ve permeado por la correlación comidas-servicio del vino, y aunque para los cubanos se evidencia falta de opinión por desconocimiento, el número de abstinencia no es tampoco señal de calidad y satisfacción por los servicios ofrecidos. 
Resultados de la aplicación de la Matriz DAFO. Análisis de los factores externos e internos.

El restaurante tiene un nivel de desarrollo elevado, sus productos y servicios tienen calidad aceptable y mercado seguro, deben aplicar y priorizar las estrategias ofensivas que le permitan maximizar sus fortalezas para aprovechar aún más las oportunidades del mercado, se recomienda aplicar estrategias de marketing integrativas y de crecimiento, debe ser actualizado el plan de ventas e implementar los programas de capacitación de los trabajadores en el servicio de vinos que permita su integración a los productos ofertados, para que sea percibido como un elemento clave y elevar los estándares de calidad del restaurante.

Propuesta de plan de mejora para el servicio del vino según fundamentos metodológicos: segunda fase.

Esta segunda fase 0 momento metodológico, que tiene implícito también determinados pasos, trae aparejada las siguientes consideraciones. 1ro. - Que todo plan de mejora es una planificación táctica para incidir que los estándares de calidad de un producto o servicio superen su nivel.

2do.- Que, aunque parte de la fotografía de un momento, o situación dada, todo plan es dinámico, movible; no puede perder su pertinencia, es algo que se elabora a la medida.

3ro. - Que no se abstrae de la realidad, sino que se nutre de ella.

4to.- Que implica costes, pero también beneficios y que una vez colocados en la balanza son factores que se deben controlar, reajustar.

5to.- Que han de diseñarse para que se apliquen no para tener un grupo de acciones plasmadas en un papel.

6to.- Que debe tener un grado de implicación personal para cada miembro de una empresa, no es un plan para que sólo le interese al superior.

Los autores, partiendo de estos elementos escogen los siguientes pasos para elaborar el plan: 

a) Delimitación de los objetivos
si asisten en familias 0 independientes.

b) Descripción de los problemas que se pretenden reducir por áreas de acción

c) Identificación de las relaciones de dependencia entre las acciones, las características de la empresa y mejoras potenciales

d) Delimitación de las acciones en términos de plazos y control

e) Confección final del plan

f) Discusión de la propuesta con la administración de la instalación.

Propuesta de plan de mejora para el servicio de vinos en el restaurante no estatal "EI Paso"

\section{1 - Área clave: Mercado.}

Problema a resolver: Delimitación del mercado que frecuenta la instalación.

Acciones a desarrollar:

- Establecer un registro diario de clientes para análisis de comportamiento

- Confección de plantilla que incluya: edad, sexo, nacionalidad,

Problema a resolver: Insuficiente conocimiento respecto a gustos y preferencias de estos mercados.

Acciones a desarrollar:

- Elaborar encuestas de satisfacción.

- Realizar muestreo de pedidos de alimentos y vinos para delimitar preferencias.

Problema a resolver: Desconocimiento de aspectos relacionados con los mercados emisores: comportamientos de arribos territoriales, nacionales, vínculos con el turismo de ciudad, entre otras.

Acciones a desarrollar: desarrollar acciones de capacitación, coordinar en temporadas bajas para que apoyo a esta capacitación.

\section{2 - Área clave: Servicios}

Problema a resolver: Servicio de vinos deficiente, sin especialización.

Acciones a desarrollar: implementar el servicio especializado de vinos una vez recibida la capacitación y 
cumplida la demanda de requerimientos.

Problema a resolver: Dificultades con el aprovisionamiento.

\section{Gestionar posibles} contrataciones amparadas en el aparato legal vigente.

Problema a resolver: Poca gestión de venta.

Acciones a desarrollar: aplicar técnicas de ventas: merchandising, ventas personales, desarrollar acciones de capacitación a través de CCM.

\section{3 - Área clave: Suministros.}

Problema a resolver: Insuficientes insumos para realizar los servicios de vinos.

Acciones a desarrollar: compra de insumos (stock de vinos y otros accesorios)

Problema a resolver: Ausencia de instrumentos requeridos para el servicio de vinos.

Acciones a desarrollar: compra de instrumentos requeridos para el servicio especializado de vinos.
Problema a resolver: Carencia de expositores.

Acciones a desarrollar: sustitución de estantería, creación de expositores.

Problema a resolver: Ausencia de suministradores estables de vinos.

Acciones a desarrollar: gestionar posibles contrataciones amparadas en el aparato legal a fin de disminuir el encarecimiento de productos.

4 - Área clave: Inversiones.

Problema a resolver: Necesidad de reestructurar locales.

Acciones a desarrollar:

- Construcción de la cava.

- Coordinar la ejecución de la obra con el agente constructor no estatal.

Problema a resolver: Falta de armonía en la decoración.

Acciones a desarrollar: mejorar la decoración con ajuste al tema.

Problema a resolver: Demoras en las contrataciones por carencia de personal capacitado en servicios especializados. 
Acciones a desarrollar: gestionar contratación con terceros: ejemplo diseñadores, arquitectos, artistas plásticos

\section{5 - Área clave: Capacitación.}

Problema a resolver: Insuficiente preparación de los dependientes gastronómicos.

Acciones a desarrollar: solicitar Curso Sommelier, solicitar Cursos sobre Técnicas de venta merchandising sobre la base de previas coordinaciones con el CCM.

Problema a resolver: Baja cultura sobre el vino en general y sus posibilidades de empleos.

Acciones a desarrollar: solicitar seminarios y talleres sobre generalidades del vino.

Problema a resolver: Insuficiente conocimiento de las normas cubanas que rigen la restauración.

Acciones a desarrollar: - Seminariar sobre la NC: 126 y 127.

Leyenda: - CCM: Centro de capacitación del MINTUR (Antiguo Formatur)

- AAVV: Agencias de Viajes.

\section{Conclusiones}

Fueron identificadas insuficiencias en el restaurante "El Paso" de la ciudad de Camagüey, respecto al conocimiento del vino y sus posibilidades de integración como servicio, para satisfacer los gustos y preferencias del cliente, lo que posibilitó elaborar un plan de mejoras en el servicio de vinos que permita su incorporación a los productos ofertados, la metodología utilizada permitió incidir de forma precisa en la implementación de mejoras para lograr la incorporación gradual, pautada, orgánica y procesual del servicio especializado de vinos en este restaurante, percibido ahora como un elemento clave para elevar los estándares de calidad del restaurante, a estimarse por los criterios de satisfacción que se obtengan de los clientes que lo visitan.

\section{Bibliografía}

Aguilar, J. (2016). Proceso de mejora continua. Obtenido de http://www.conductitlan.net/p sicologia_organizacional/la_ mejora_continua.pdf 
Boné, D., Rey, A., Fusté, F. (2015). Los hoteles temáticos: un caso descriptivo de creación de Experiencias a través de ejemplos singulares españoles. Papers de Turisme, (58), 47- Obtenido de:

http://www.papersdeturisme.g va.es/ojs/index.php/Papers/ar ticle/download/448/377

Carvache, M., Carvache, W., Orden, M., Macas, C. (2017). Satisfacción y motivación de la demanda gastronómica en General. Revista Iberoamericana de Turismo, 68-84, Recuperado de: http://www.progep.ufal.br/see r/index.php/ritur/article/view/2 $710 / 2426$

Castro, Y. (2015). Artículo: Aportar al desarrollo del comercio cubano. Periódico Granma, 23-05-2015, edición No.123

Monroy, M., Urcádiz, F. (2018). Calidad en el servicio y su incidencia en la satisfacción del comensal en restaurantes de La Paz, México. Revista Investigación Administrativa, 48(123), 71-91. e-ISSN: 2448-7678. Recuperado de: http://www.scielo.org.mx/sciel o.php?pid=S2448$76782019000100006 \&$ script $=$ sci_abstract.

Normas Internacionales, ISO.9000 (2016). Obtenido de http://www.normas $9000 . c o m / i$ so-9000-59.html
Parra, C., Vasallo, Y. (2016). El concepto del nuevo servicio y su aplicación en el diseño de procesos servicio de restauración. Ibarra, Ecuador, Universidad Técnica del Norte.

Rentería, S. (2017). Calidad de servicio frente a posibles problemas con los clientes. Restaurante "Rosso Pastas y Vinos" en la ciudad de Machala. Machala, Ecuador. Obtenido de http://www.scielo.org.ar/scielo .php?script=sci_arttext\&pid= S1851-17322012000300006

Sánchez, K. (2012). "El turismo como fenómeno social complejo", Vol.5, No13. Obtenido de http://www.eumed.net/rev/tur ydes/13/klso.html 